\title{
Keyword Surveillance and the Development of Evolving Reporting in Austin, Texas
}

\author{
Ashley N. Hawes \\ Austin Public Health, Austin, Texas, United States
}

Objective

Austin Public Health's Public Health Emergency Preparedness program utilizes a variety of tools and resources to create informative, event-specific, and engaging syndromic surveillance reports to share 1) internally within Austin Public Health; 2) with City of Austin and Travis County partners; 3) local health care coalition members; and 4) the public during events that affect the Austin, Texas metropolitan area.

\section{Introduction}

Austin Public Health creates a variety of syndromic surveillance reports for events throughout the Austin, Texas metropolitan area. These events range from responses to major disasters such as the 2017 Hurricane Harvey sheltering to ongoing special event monitoring such as University of Texas football games and the Austin City Limits music festival. Partnerships within the Austin metropolitan region are crucial to ensuring the information-sharing necessary to create robust reports, as well as during the followup process of requesting feedback from partners on the usefulness of the reports. Austin Public Health's Public Health Emergency Preparedness program utilizes a variety of tools and resources to create informative, event-specific, and engaging reports, fulfilling multiple reporting needs for all partners.

\section{Methods}

The process of generating syndromic surveillance reports begins by keyword surveillance of hospital emergency room chief complaint data. Keywords are keyed into the Austin metropolitan area's hospital free-text chief complaints via the Capital Area Public Health and Medical Coalition. The searchable keywords are queried to create a baseline picture of an evolving event. Data are also requested and gathered from multiple partners including local news stations, the National Weather Service, the City of Austin's Office of Vital Records (birth and death certificates), social media platforms, Austin 3-1-1, and Austin/Travis County Emergency Medical Services. All data are then analyzed, visualized and displayed in reports that are distributed via multiple platforms including email, social media, governmental websites, Geographic Information System (GIS) storymaps, and WebEOC. Reports are then combined into event end summaries. Accompanying the final summary report are feedback surveys.

\section{Results}

The ability to request keywords in an open communication pathway between hospitals, the Capital Area Public Health and Medical Coalition, and the local health department has bolstered area partnerships. Previous surveillance reports have been reported to be both useful and beneficial to departmental, community and health coalition partners. For example, the 2017 report following Hurricane Harvey was used by local hospitals for planning staffing and surge needs, and the 2018 heat report is being used to determine the placement of future cooling stations at special events. A 2019 surveillance report on dockless scooter injuries will be used to inform risk factors and trauma injury severity. Requested changes from partners have included: the addition of graphs, keyword-specific changes, inclusion of social media and broadcast media data, and the use of information from other partners to create a final event or year-end summary report.

\section{Conclusions}

Keyword surveillance of hospital chief complaint data and of other local real-time data are innovative tools to creating meaningful syndromic surveillance reports that provide situational awareness and are adaptable to the needs of events and situations in the area. The development and evolution of these syndromic surveillance reports has helped to build a rapidly deployable syndromic surveillance system that can provide key data for preparing for and responding to future disaster events. By engaging local and regional partners in an iterative process for developing these reports, APH ensures ongoing improvement, thereby providing more powerful and useful reports to all partners involved.

SDS Annual Conference Proceedings 2019. This is an Open Access article distributed under the terms of the Creative Commons AttributionNoncommercial 4.0 Unported License (http://creativecommons.org/licenses/by-nc/3.0/), permitting all non-commercial use, distribution, and reproduction in any medium, provided the original work is properly cited. 


\section{Acknowledgement}

Austin Public Health, City of Austin, Travis County, Capital Area Public Health and Medical Preparedness Coalition

Austin Public Health
$\begin{aligned} & \text { Austin } \\ & \text { Public } \\ & \text { Health } \\ & \text { HEAT-RELATED ILLNESS AND MORTALITY SURVEILLANCE }\end{aligned}$
Background
Austin Public Health initiated heat-related illness surveillance on May 15, 2018 and will continue through
September 30, 2018. The primary data sources are: (1) emergency department (ED) chief complaint data from 15
Austin Metro Area hospitals and (2) City of Austin mortality records. Two sources are utilized for daily heat index,
the National Weather Service (NWS) National Oceanic Atmospheric Association (NOAA) and the lowa Environmental
Mesonet at lowa State University. This report provides summary findings for this data. Please note that ED Chief
Complaints are pre-diagnostic. For additional details regarding methodology or incident definitions, please contact
Ashley Hawes at Ashlev. Hawes@austintexas.gov

Monitoring Period Summary

May 1, 2018 - September 23, 2018

\begin{tabular}{l|l}
\hline Heat Advisory/Warning & 2 Heat Advisory, 1 Excessive Heat Warning \\
Heat-Related Deaths & 4 \\
Heat-Related IIIness & 819 including dehydration, 283 excluding dehydration \\
\hline Drowning Information & 6 deaths, 30 related ED visits \\
\hline
\end{tabular}

\begin{tabular}{|c|c|c|c|c|}
\hline & & \multicolumn{2}{|c|}{ Count of ED Visits by Gender } & \\
\hline \multicolumn{2}{|c|}{$\begin{array}{l}\text { Number of Visits by Zip Code } \\
(20+\text { visits shown) }\end{array}$} & \multirow{2}{*}{$\begin{array}{l}\text { Female } \\
\text { Male } \\
\end{array}$} & \multirow{2}{*}{$\begin{array}{l}352 \\
467\end{array}$} & \\
\hline 78610 & 24 & & & \\
\hline 78626 & 25 & Total & 819 & \\
\hline 78640 & 45 & & & \\
\hline 78660 & 31 & May 1,2 & eptem & \\
\hline 78664 & 24 & & & \\
\hline 78701 & 25 & & & $\begin{array}{l}0.18 \\
: 19-29\end{array}$ \\
\hline 78704 & 20 & & & $-30-39$ \\
\hline 78745 & 24 & & & .50 .59 \\
\hline 78753 & 37 & & & $=60.69$ \\
\hline 78758 & 48 & $103,13 \%$ & & \\
\hline
\end{tabular}

Figure 1. Example of page 1 of 2018 Heat Report sent out by Austin Public Health to partners 